EPJ Web of Conferences 41, 05004 (2013)

DOI: $10.1051 /$ epjconf/20134105004

C) Owned by the authors, published by EDP Sciences, 2013

\title{
Femtosecond Mid-Infrared Study of the Aqueous Solution Photochemistry of a CO-Releasing Molecule (CORM)
}

\author{
P. Rudolf ${ }^{1}$, F. Kanal ${ }^{1}$, D. Gehrig ${ }^{1}$, J. Niesel ${ }^{2}$, T. Brixner ${ }^{1}$, U. Schatzschneider ${ }^{2}$, and P. Nuernberger ${ }^{1 \text { a }}$ \\ 1 Institut für Physikalische und Theoretische Chemie, Universität Würzburg, Am Hubland, \\ 97074 Würzburg, Germany \\ 2 Institut für Anorganische Chemie, Universität Würzburg, Am Hubland, 97074 Würzburg, \\ Germany
}

\begin{abstract}
Ultraviolet irradiation of CO-releasing molecules (CORMs) in water eventually leads to the loss of several carbon monoxide ligands. We show for an exemplary manganese tricarbonyl CORM that only one ligand is photolyzed off on an ultrafast timescale and that some molecules may undergo geminate recombination.
\end{abstract}

\section{Introduction}

In recent years, biological applications of CO-releasing molecules (CORMs), which may be utilized as an in-situ source for carbon monoxide, an endogenous gasotransmitter in the human body, have been demonstrated. Suitable compounds have to be stable in air and in water, soluble in water, and specific to particular tissues. Recently synthesized metal carbonyl complexes can act as dark-stable prodrugs under physiological conditions $[1,2]$. One of them is the $\left[\mathrm{Mn}(\mathrm{CO})_{3}(\mathrm{tpm})\right] \mathrm{Cl}$ (tris(pyrazolyl)methane (tpm) manganese tricarbonyl) complex (Fig. 1a) investigated in this study. The complex undergoes photoinduced $\mathrm{CO}$ release upon UV irradiation and has been shown to be cytotoxic against cancer cells [2].

Whereas the ultrafast dynamics of CO liberation in water-insoluble metal carbonyls has been studied for many compounds (e.g. in [4-6]), the photolysis of CO in water-soluble CORMs with suitable properties for biological applications have so far only been studied on a macroscopic scale and at irradiation times of several minutes. For the CORM studied here, UV irradiation eventually leads to the release of two [2] or even all three $\mathrm{CO}$ ligands [3]. We have studied the primary processes of $\mathrm{CO}$ release from the CORM using UV/mid-IR transient absorption spectroscopy (for details on the experimental setup, cf. [7]) with two different pump wavelengths supported by linear spectroscopy methods and TDDFT calculations.

\section{Results and Discussion}

The CORM under study exhibits UV absorption bands at $270 \mathrm{~nm}$ and $350 \mathrm{~nm}$ (Fig. 1b). Excitation at $270 \mathrm{~nm}$ leads to more excess energy than at $350 \mathrm{~nm}$, but also the orbitals involved indicate that the ultrafast CO liberation might differ: TDDFT calculations show that a transition from an orbital located at the manganese atom to an orbital which has contributions delocalized over the three CO ligands takes part in the lower-energy band.

\footnotetext{
${ }^{\text {a }}$ e-mail: nuernberger@phys-chemie.uni-wuerzburg.de
}

This is an Open Access article distributed under the terms of the Creative Commons Attribution License 2.0, which permits unrestricted use, distribution, and reproduction in any medium, provided the original work is properly cited. 

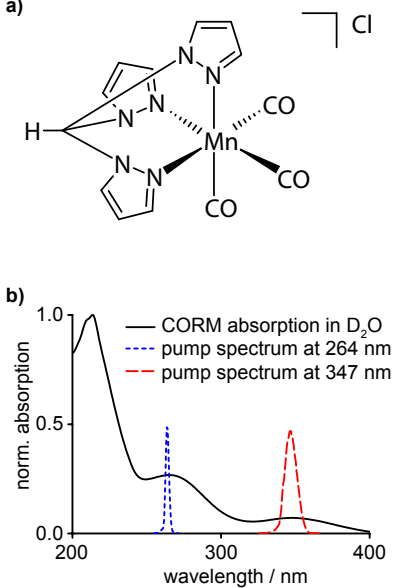

c)

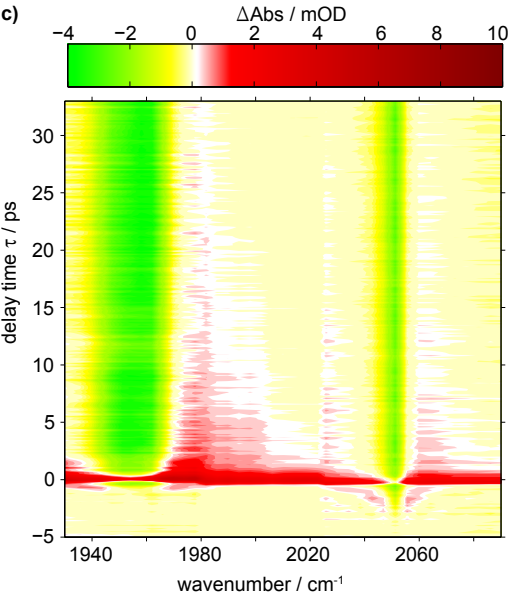

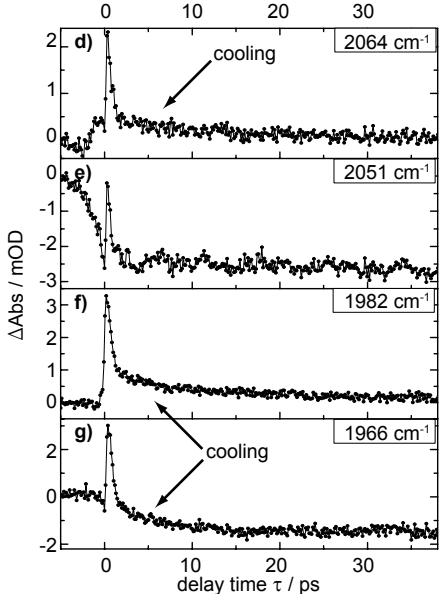

Fig. 1. a) The studied CO-releasing molecule (CORM). b) CORM absorption spectrum and the spectra of the two UV pump pulses used. c) Transient absorption recorded with $264 \mathrm{~nm}$ pump pulses and mid-IR probe pulses. d-g) Transient signals at selected wavenumbers, extracted from c). Note that signals at $\tau<0$ are due to perturbed free induction decay, whereas the positive spikes for small positive values of $\tau$ are due to solvated electron dynamics originating from a two-photon process [8].

\subsection{Amount of ligands initially photolyzed}

When excited with pump pulses at $264 \mathrm{~nm}$, the mid-IR transient absorption exhibits two distinct ground-state bleaches (Fig. 1c) around $1958 \mathrm{~cm}^{-1}$ (see also the transient absorption signal at a probe wavenumber of $1966 \mathrm{~cm}^{-1}$ in Fig. $1 \mathrm{~g}$ ) and $2051 \mathrm{~cm}^{-1}$ (Fig. 1e), which agree with FTIR measurements of the compound in $\mathrm{D}_{2} \mathrm{O}$ solution. Since they persist for long delay times, they evidence ultrafast $\mathrm{CO}$ liberation. Note that very similar behavior is observed after excitation with pump pulses at $347 \mathrm{~nm}$.

Two new absorption bands appear at around $1980 \mathrm{~cm}^{-1}$ (Fig. 1f) and $1857 \mathrm{~cm}^{-1}$ (Fig. 2b), which directly show the formation of a dicarbonyl species [3,9], i.e. only one CO ligand has been photolyzed on an ultrafast timescale. The dicarbonyl signal is constant over at least 100 ps (Fig. 2b), suggesting that a stable dicarbonyl has formed.

\subsection{Vibrational excess energy}

The energy of the UV photon is much higher than the energy needed to cleave the Mn-CO bond, leaving energy in the vibrational modes of the molecules which in theory is sufficient for several quanta of the CO stretch mode. Whereas it has been found that UV excitation of hexacarbonyls leads to a significant amount of molecules with vibrational hot bands in the $\mathrm{CO}$ stretch [4], that has not been observed in a study on dicarbonyls [5]. In the data of our CORM, no distinct hot bands are observed, hence the $\mathrm{CO}$ stretch of the photoproduct is not excited in the course of the photolysis.

Therefore, the excess energy has to go to low-frequency modes of the photoproduct. Before vibrational relaxation is over, these modes couple to the CO stretch [4] of the newly-formed dicarbonyl, detectable as broadened absorption which narrows upon cooling. By probing slightly shifted from the absorption band center, this cooling within $10 \mathrm{ps}$ is seen for both product bands and is shown for the lower energy vibration in Fig. 2c.

\subsection{Quantum yield of dicarbonyl formation}

If each UV excitation of the CORM leads to photodissociation of one $\mathrm{CO}$, this does not necessarily mean that it always leads to $\mathrm{CO}$ liberation and the formation of a dicarbonyl. Geminate recombination 

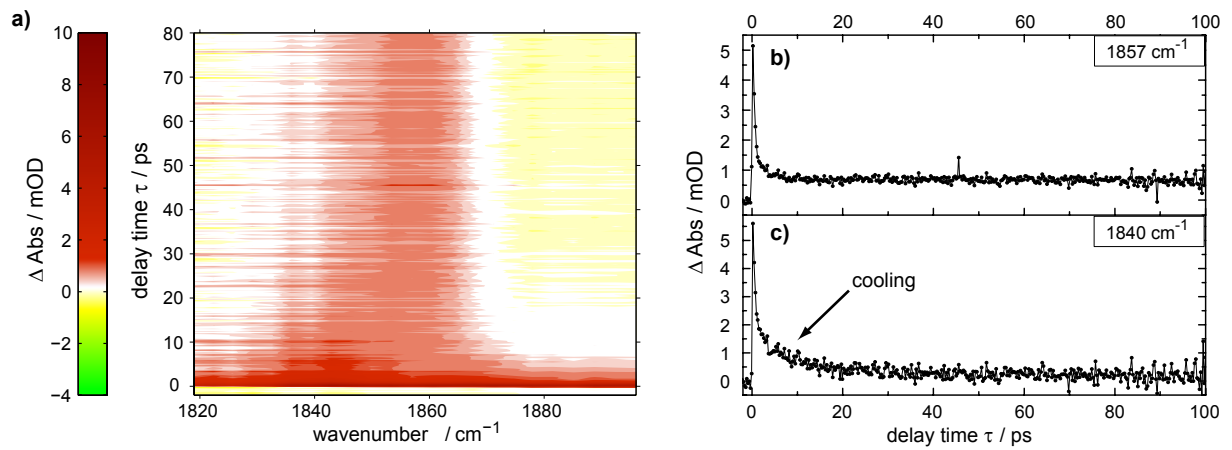

Fig. 2. a) Transient absorption for the region of the dicarbonyl product absorption recorded with $347 \mathrm{~nm}$ pump pulses and mid-IR probe pulses. b-c) Transient signals at selected wavenumbers, extracted from a).

may restore the initial CORM by rebinding the photolyzed $\mathrm{CO}$. Measurements for $\mathrm{Cr}(\mathrm{CO})_{6}$ in alkanes have shown that this becomes less likely the smaller the solvent molecules are [6].

The dicarbonyl species does not show an IR absorption above $2000 \mathrm{~cm}^{-1}$, but the intact CORM absorbs around $2051 \mathrm{~cm}^{-1}$. When geminate recombination occurs, the coupling of the $\mathrm{CO}$ band to the low-frequency modes also leads to a broadening of the absorption at $2051 \mathrm{~cm}^{-1}$ as long as the CORM is vibrationally hot. This is therefore seen as a positive signal (due to the broadened absorption line of recombined CORM) around the negative ground-state bleach (caused by CORM molecules that lost a CO). The data at $2064 \mathrm{~cm}^{-1}$ (Fig. 1d) shows this contribution which disappears when cooling is finished.

\section{Conclusion}

UV excitation of a CO-releasing molecule in aqueous solution leads to ultrafast photolysis of one $\mathrm{CO}$ ligand. Geminate recombination may occur, but predominantly the photolyzed $\mathrm{CO}$ is liberated and the vacant coordination site might be filled by an incoming solvent molecule. Excess energy is transferred to low-frequency vibrational modes, the remaining $\mathrm{CO}$ ligands are not vibrationally excited through the UV excitation of the CORM. Further loss of CO most likely necessitates manganese oxidation or another interaction with light. The elucidation of the primary processes presented here is essential for a full understanding of the biological activity of CORMs.

\section{References}

1. R. Alberto and R. Motterlini, Dalton Trans. 1651-1660 (2007)

2. J. Niesel, A. Pinto, H. W. Peindy N'Dongo, K. Merz, I. Ott, R. Gust, and U. Schatzschneider, Chem. Comm. 1798-1800 (2008)

3. H.-M. Berends and P. Kurz, Inorg. Chim. Acta 380, 141-147 (2012)

4. T. P. Dougherty and E. J. Heilweil, Chem. Phys. Lett. 227, 19-25 (1994)

5. T. P. Dougherty and E. J. Heilweil, J. Chem. Phys. 100, 4006-4009 (1994)

6. T. Lian, S. E. Bromberg, M. C. Asplund, H. Yang, and C. B. Harris, J. Phys. Chem. 100, 11994-12001 (1996)

7. P. Rudolf, J. Buback, J. Aulbach, P. Nuernberger, and T. Brixner, J. Am. Chem. Soc. 132, 15213-15222 (2010)

8. R. Laenen, T. Roth, and A. Laubereau, Phys. Rev. Lett. 85, 50-53 (2000)

9. W. Huber, R. Linder, J. Niesel, U. Schatzschneider, B. Spingler, and P. C. Kunz, Eur. J. Inorg. Chem. 3140-3146 (2012) 